\title{
䌼 Springer
}

Draft Manuscript for Review

\section{Modelle für die Berechnung der Ausziehtragfähigkeit von selbstbohrenden Holzschrauben}

\begin{tabular}{|r|l|}
\hline Journal: & Holz als Roh- und Werkstoff \\
\hline Manuscript ID: & HRW-09-0003.R1 \\
\hline Manuscript Type: & ORIGINALARBEITEN / ORIGINALS \\
\hline Author: & 24-Apr-2009 \\
\hline Complete List of Authors: & $\begin{array}{l}\text { Frese, Matthias; Universität Karlsruhe, Lehrstuhl für } \\
\text { Ingenieurholzbau und Baukonstruktionen } \\
\text { Fellmoser, Peter; Blaß \& Eberhart Ingenieurbüro für } \\
\text { Baukonstruktionen } \\
\text { Blaß, Hans Joachim; Universität Karlsruhe, Lehrstuhl für } \\
\text { Ingenieurholzbau und Baukonstruktionen }\end{array}$ \\
\hline Keywords: & $\begin{array}{l}\text { wood screw, self-tapping screw, self-drilling screw, inclined screw, } \\
\text { withdrawal capacity, withdrawal resistance, withdrawal parameter }\end{array}$ \\
\hline
\end{tabular}




\title{
Modelle für die Berechnung der Ausziehtragfähigkeit von selbstbohrenden Holzschrauben
}

\author{
M. Frese (Korrespondenzautor) \\ Universität Karlsruhe, Lehrstuhl für Ingenieurholzbau und Baukonstruktionen, \\ Kaiserstraße 12, 76131 Karlsruhe, Germany \\ Matthias.Frese@holz.uka.de \\ P. Fellmoser \\ Blaß \& Eberhart \\ Ingenieurbüro für Baukonstruktionen \\ Pforzheimer Straße 15b, 76227 Karlsruhe, Germany \\ H.J. Blaß \\ Universität Karlsruhe, Lehrstuhl für Ingenieurholzbau und Baukonstruktionen, \\ Kaiserstraße 12, 76131 Karlsruhe, Germany
}


Zusammenfassung Berechnungsgleichungen für die Ausziehtragfähigkeit von selbstbohrenden Holzschrauben in Nadelholz wurden hergeleitet. Auf der Grundlage von Ergebnissen aus 1850 Ausziehversuchen mit unterschiedlichsten Schrauben wurden mittels Regressionsrechnungen eine Gleichung für den Ausziehwiderstand und zwei weitere für den Ausziehparameter ermittelt. Unabhängige Variable in den Gleichungen sind der Schraubennenndurchmesser, die Einschraubtiefe im Holz und der charakteristische Wert der Rohdichte des verwendeten Nadelholzes. Die Gleichungen sind gültig für eine Schraubenneigung zwischen $45^{\circ}$ und $90^{\circ}$. Die Gleichung für den Ausziehwiderstand liefert im Vergleich, auch mit herkömmlichen Gleichungen in Normen, die günstigsten Werte. Für selbstbohrende Schrauben mit geometrischen Eigenschaften, die mit denjenigen des Untersuchungsmaterials vergleichbar sind, bietet sie die Möglichkeit, den Ausziehwiderstand, genauer als bisher, rechnerisch abzuschätzen. Insofern kann der experimentelle Aufwand, der für die Zulassung von Schrauben erforderlich ist, reduziert werden.

\section{Models for the calculation of the withdrawal capacity of self-tapping screws}

Abstract Equations to calculate the withdrawal capacity of self-tapping screws, arranged in softwood, were determined. Based on results of 1850 withdrawal tests and by means of a regression analysis one equation for the withdrawal resistance and two further equations for the withdrawal parameter (resistance over nominal screw diameter and penetration depth) were derived. Nominal screw diameter, penetration depth and characteristic density of the softwood used are the independent variables in the equations, which are valid for an angle in the range of $45^{\circ}$ to $90^{\circ}$ between the screw axis and the grain.

The equation for the withdrawal resistance delivers, also in comparison with code rules, the most favourable values. It enables now a more accurate estimation of the withdrawal resistance for wood screws with geometrical properties similar to those of the screws used for the tests. Hence, the benefit of this study is the reduction in testing, which is currently necessary for technical approvals. 


\section{Einleitung}

\subsection{Allgemeines}

Die Verwendung von Holzschrauben (im Folgenden kurz „Schrauben“) hat in den vergangenen Jahren zugenommen, nicht zuletzt wegen der erfolgreichen und rasanten Weiterentwicklung von selbstbohrenden Schrauben. So ergänzen beispielsweise spezielle Schrauben für Anschlüsse zwischen Haupt- und Nebenträgern, für Querzug- und Querdruckverstärkungen sowie für Befestigungen von Aufdach-Dämmsystemen die herkömmliche Verbindungstechnik. Auf Grund der bevorzugt schrägen Anordnung von Schrauben (Abb. 1) steht nunmehr das Tragverhalten einer Schraube, die in Richtung ihrer Achse beansprucht wird, im Vordergrund, weniger dasjenige bei Beanspruchung rechtwinklig zu ihrer Achse.

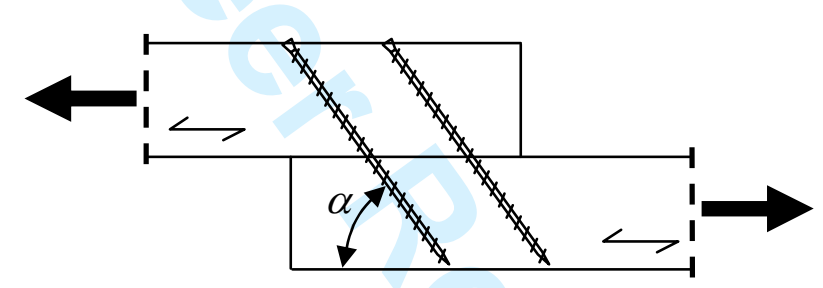

Abb. 1 Schräg, unter dem Einschraubwinkel $\alpha$ angeordnete und in Richtung der Schraubenachse beanspruchte Schrauben

Fig. 1 Self-tapping screws inserted at an angle $\alpha$ to grain and loaded in tension

Für tragende Holzverbindungen werden genormte Schrauben oder solche, deren Eignung über eine allgemeine bauaufsichtliche Zulassung ${ }^{1}$ (abZ) nachgewiesen ist, verwendet: Genormte Schrauben sind in DIN 96, DIN 97 oder DIN 571 geregelt und weisen Gewinde und Schraubenspitzen nach DIN 7998 auf. Schrauben mit abZ sind in der Regel selbstbohrende Schrauben mit Teil- oder Vollgewinde, Längen bis $600 \mathrm{~mm}$ und Nenndurchmessern bis $14 \mathrm{~mm}$. Insbesondere die Formvielfalt von selbstbohrenden Schrauben hat zu etlichen Zulassungen geführt.

\section{${ }^{1}$ National technical approval}


DIN 1052 und EN 1995-1-1 enthalten unterschiedliche Berechnungsgleichungen und Verfahren für die Ermittlung des charakteristischen Wertes des Ausziehwiderstandes $R_{a x, k}$. Dieser Wert beziffert die rechnerische Beanspruchbarkeit auf Herausziehen. Nach DIN 1052 muss eine Schraube zunächst in eine von drei Tragfähigkeitsklassen (TFK) eingestuft sein. Mit der Einstufung ist der charakteristische Wert des Ausziehparameters (in DIN 1052: $f_{1, k}$ ) berechenbar und dann der Ausziehwiderstand der Schrauben. Der Ausziehparameter ist aus experimenteller Sicht eine auf Einschraubtiefe und Nenndurchmesser bezogene maximale Ausziehkraft. In EN 1995-1-1 kann der charakteristische Wert des Ausziehparameters (darin: $f_{a x, k}$ ) bereits direkt berechnet werden oder er ist in Sonderfällen durch Ausziehversuche gemäß EN 14592 zu ermitteln. Sodann lässt sich der Ausziehwiderstand berechnen. Beide Verfahren in DIN 1052 bzw. EN 1995-1-1 erforderten in der Vergangenheit, wie auch noch heute, einen großen Prüfaufwand.

Es war daher das Ziel dieser Untersuchung, den Ausziehwiderstand bzw. -parameter selbstbohrender Schrauben herstellerunabhängig in Form von Berechnungsmodellen anzugeben. Der Nutzen dieser Berechnungsmodelle liegt darin, dass Schrauben, für die solche Modelle gültig sind, hinsichtlich der Ausziehtragfähigkeit nicht mehr im sonst üblichen Umfang geprüft werden müssten.

Auf Grund einer mittlerweile stark angewachsenen Datensammlung von Versuchsergebnissen aus Zulassungsverfahren und Forschungsarbeiten erschien es nutzbringend, den Ausziehwiderstand direkt oder mittelbar über den Ausziehparameter neu zu modellieren. Ein neues Modell wäre damit an umfangreiche aktuelle Versuchsergebnisse angepasst und besäße eine hohe Allgemeingültigkeit, da Schraubentypen unterschiedlichster Hersteller in der Datensammlung anteilig erfasst sind.

Dieser Beitrag beschreibt alle wesentlichen Schritte der Modellierung bis hin zu Berechnungsgleichungen. Diese Gleichungen (oder Modelle) sind abhängig von der Einschraubtiefe $\ell_{e f}$, dem Nenndurchmesser $d$ und der charakteristischen Rohdichte $\rho_{k}$ (einer gewählten Nadelholz-Festigkeitsklasse aus EN 338). Sie sind für Einschraubwinkel $\alpha$ zwischen $45^{\circ}$ und $90^{\circ}$ anwendbar. Es wird schließlich gezeigt, dass gegenwärtig gültige Berechnungsansätze in baupraktisch relevanten Fällen zu völlig unterschiedlichen Ergebnissen führen können. Daher möchte der 
Beitrag auch dazu anstoßen, die gegenwärtig praktizierte Berechnung der Ausziehtragfähigkeit zu überdenken.

\subsection{Stand der Technik}

Im Vergleich zu genormten Schrauben besitzen selbstbohrende Schrauben mit abZ folgende Vorteile bzw. Eigenschaften:

- Das $d_{k} / d$-Verhältnis ist geringer, im Mittel beträgt es etwa 0,65 (Abb. 2), bei Schrauben mit Gewinde nach DIN 7998 im Mittel 0,70. Die profilierte Ausbildung des Gewindebereichs mit großem Flankenflächenanteil ermöglicht eine hohe Kraftübertragung ins umgebende Holz.

- Sie werden in der Regel nach dem Aufrollen des Gewindes gehärtet. Das Fließmoment, die Torsionsfestigkeit und die Zugtragfähigkeit werden dadurch deutlich gesteigert. Die Steifigkeit der Verbindung nimmt zu, die Gefahr des „Abdrehens“ ab. Sie können daher ohne Vorbohren eingedreht werden.

Um diese Vorteile bzw. Eigenschaften in baupraktischen Fragestellungen zu nutzen, wurden in der Vergangenheit viele Untersuchungen durchgeführt und entsprechende Berechnungsansätze veröffentlicht (Schrauben in spaltgefährdeten Verbindungen (Blaß und Schmid 2001, Bejtka und Blaß 2005), Verbindungen mit geneigt oder gekreuzt angeordneten Schrauben (Kevarinmäki 2002, Bejtka und Blaß 2002) sowie Querzug- und Querdruckverstärkungen (Jönsson 2005, Bejtka und Blaß 2006)).

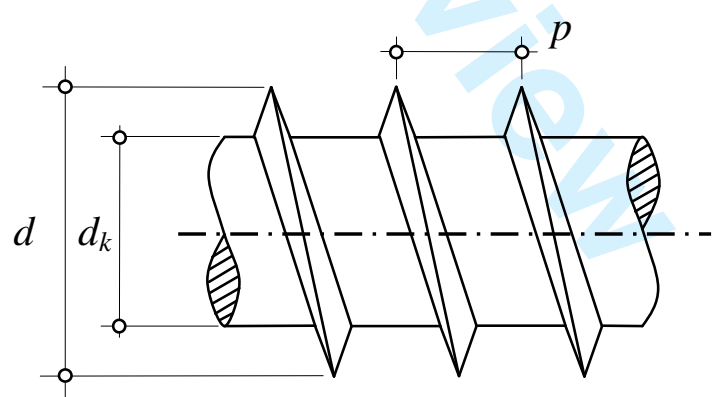

Abb. 2 Detail eines Schraubengewindes: Bezeichnungen Nenndurchmesser $d$, GewindeInnendurchmesser $d_{k}$ und Gewindesteigung $p$

Fig. 2 Detail of a screw thread: denotations nominal diameter $d$ (= nominal screw size), threadroot diameter $d_{k}$ and thread pitch $p$

Folgende Berechnungsgleichungen (aus Normen und dem Schrifttum) für den charakteristischen Ausziehwiderstand $R_{a x, k}($ in N) seien kurz vorgestellt; der Kopfdurchziehwiderstand wird in den Gleichungen nicht berücksichtigt: 


$$
\begin{aligned}
& R_{a x, k}=\frac{f_{1, k} \cdot d \cdot \ell_{e f}}{\sin ^{2} \alpha+\frac{4}{3} \cdot \cos ^{2} \alpha} \quad\left(45^{\circ} \leq \alpha \leq 90^{\circ}\right), \\
& R_{a x, k}=\frac{0,52 \cdot d^{0,5} \cdot \ell_{e f}^{0,9} \cdot \rho_{k}^{0,8}}{1,2 \cdot \cos ^{2} \alpha+\sin ^{2} \alpha} \quad\left(0^{\circ} \leq \alpha \leq 90^{\circ}\right), \\
& R_{a x, k}=\frac{n_{e f} \cdot f_{a x, k} \cdot d \cdot \ell_{e f} \cdot k_{d}}{1,2 \cdot \cos ^{2} \alpha+\sin ^{2} \alpha} \quad\left(\begin{array}{c}
6 \mathrm{~mm} \leq d \leq 12 \mathrm{~mm} \\
0,60 \leq d_{k} / d \leq 0,75 \\
\alpha \geq 30^{\circ}
\end{array}\right) .
\end{aligned}
$$

Darin sind $f_{1, k}$ und $f_{a x, k}$ in $\mathrm{MPa}, d$ und $\ell_{e f}$ in mm und $\rho_{k}$ in $\mathrm{kg} / \mathrm{m}^{3}$ einzusetzen.

Gleichung (1) nach DIN 1052 gibt den charakteristischen Ausziehwiderstand $R_{a x, k}$ in Abhängigkeit des charakteristischen Ausziehparameters $f_{1, k}$, des

Nenndurchmessers $d$, der Einschraubtiefe $\ell_{e f}$ und des Einschraubwinkels $\alpha$ an. Die Variable $f_{1, k}$ (in MPa), von der Tragfähigkeitsklasse abhängig, wird mit der charakteristischen Rohdichte berechnet:

TFK $1: f_{1, k}=60 \cdot 10^{-6} \cdot \rho_{k}^{2}$

TFK $2: f_{1, k}=70 \cdot 10^{-6} \cdot \rho_{k}^{2}$.

TFK $3: f_{1, k}=80 \cdot 10^{-6} \cdot \rho_{k}^{2}$

Die charakteristische Rohdichte ist in $\mathrm{kg} / \mathrm{m}^{3}$ einzusetzen, jedoch höchstens mit $500 \mathrm{~kg} / \mathrm{m}^{3}$. Die Schrauben werden mittels Prüfung nach EN 1382 bzw. in Anlehnung an DIN 1052, Anhang C in Tragfähigkeitsklassen eingestuft.

Blaß et al. 2006 werteten etwa 800 Ausziehversuche aus. Sie schlagen Gleichung (2) für den charakteristischen Ausziehwiderstand vor. Diese Gleichung wurde zur Grundlage für die Regelung in EN 1995-1-1. Darin wird der Ausziehwiderstand mittels Gleichung (3) berechnet. Es wird zusätzlich die Anzahl Schrauben $n$ einer axial beanspruchten Gruppe von Schrauben berücksichtigt. Für Schrauben mit Nenndurchmessern zwischen 6 und $8 \mathrm{~mm}$ wird die Ausziehtragfähigkeit mit dem dimensionslosen Faktor $k_{d}$ reduziert. Die Variablen $n_{e f}, f_{a x, k}$ (in MPa) und $k_{d}$ in (3) werden berechnet mit

$$
\begin{aligned}
& n_{e f}=n^{0,9}, \\
& f_{a x, k}=0,52 \cdot d^{-0,5} \cdot \ell_{e f}^{-0,1} \cdot \rho_{k}^{0,8},
\end{aligned}
$$




$$
k_{d}=\min \left\{\begin{array}{c}
d / 8 \\
1
\end{array} .\right.
$$

Die Einheit von $d$ und $\ell_{e f}$ ist mm, von $\rho_{k} \mathrm{~kg} / \mathrm{m}^{3}$. Für $d_{k} / d$-Verhältnisse außerhalb von $[0,60 ; 0,75]$ gibt es in EN 1995-1-1 eine von Gleichung (3) abweichende Variante. Diese ist gültig für einen experimentell ermittelten Ausziehparameter $f_{a x, k}$.

\section{Modellierung}

\subsection{Datensammlung und Festlegung der Datenbasis}

Die Datensammlung des Lehrstuhls für Ingenieurholzbau und Baukonstruktionen umfasst über 2400 Datensätze von Ausziehversuchen (Abb. 3). Die Datensätze enthalten Beobachtungen, nicht immer vollständig, zu den folgenden Merkmalen:

1 Einschraubwinkel

2 Nenndurchmesser

3 Gewinde-Innendurchmesser

4 Gewindesteigung

5 Einschraubtiefe

6 Prüfkörperrohdichte

7 Ausziehwiderstand

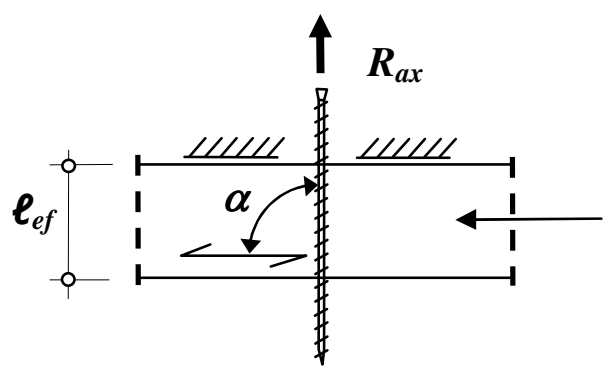

Abb. 3 Schrauben-Ausziehversuch, schematisch

Fig. 3 Schematic of the screw withdrawal test $\alpha$

$d$,

$d_{k}$,

$p$,

$\ell_{e f}$

$\rho$ und

$R_{a x}$.
Nadelholz-Prüfkörper

mit Rohdichte $\rho$ 
Eine multiple Regression (von $R_{a x}$ auf die Merkmale 1 bis 6) im Vorfeld zeigte, dass die Gewindesteigung $p$ keinen nennenswerten Einfluss auf den Ausziehwiderstand hat. Bei den untersuchten Schrauben liegt $p$ zwischen 2 und 8 mm, im Mittel bei 4,4 mm. Sie wurde daher bei der Festlegung der Datenbasis für die Modellansätze nicht berücksichtigt. Auch, um dem grundsätzlich unterschiedlichen Tragmechanismus zwischen faserparallel eingedrehten Schrauben und solchen mit Einschraubwinkeln zwischen $45^{\circ}$ und $90^{\circ}$ gerecht zu werden, enthält die Datenbasis nur Beobachtungen mit Einschraubwinkeln größer gleich $45^{\circ}$. Mit diesen beiden Randbedingungen stehen von den 2400 Datensätzen insgesamt 1847 mit ebenso vielen Beobachtungen, und zwar vollständig, je Merkmal zur Verfügung. Aus statistischer Sicht ist das vor dem Hintergrund der sonst überschaubaren Proben für wissenschaftliche Untersuchungen im Holzbau ein bemerkenswerter Umfang. Die statistischen Kennwerte der Merkmale, des $d_{k} / d$-Verhältnisses und des Ausziehparameters sind in Tabelle 1 zusammengestellt. Ausgehend von dieser Datenbasis werden alle nachfolgenden statistischen Untersuchungen durchgeführt.

Tabelle $1 \quad$ Statistische Kennwerte der Merkmale

Table $1 \quad$ Statistics of the parameters recorded

\begin{tabular}{|c|c|c|c|c|c|c|c|}
\hline Zeile & $\begin{array}{c}\text { Variable } \\
\text { (Regressor) }\end{array}$ & & $\mathrm{N}$ & $\bar{x}$ & s & Min & Max \\
\hline 1 & $\alpha$ & $\circ$ & \multirow{6}{*}{1847} & 75 & 19,1 & 45 & 90 \\
\hline 2 & $d$ & $\mathrm{~mm}$ & & 8 & 2,34 & 4,00 & 14,0 \\
\hline 3 & $d_{k}$ & $\mathrm{~mm}$ & & 5,2 & 1,53 & 2,56 & 9,70 \\
\hline 4 & $\ell_{e f}$ & $\mathrm{~mm}$ & & 61 & 25,5 & 18,8 & 140 \\
\hline 5 & $\rho$ & $\mathrm{kg} / \mathrm{m}^{3}$ & & 431 & 39,7 & 325 & 602 \\
\hline \multirow[t]{2}{*}{6} & $d_{k} / d$ & - & & 0,65 & 0,0426 & 0,559 & 0,700 \\
\hline & $\begin{array}{l}\text { Abhängige } \\
\text { (Regressand) }\end{array}$ & & & & & & \\
\hline 7 & $R_{a x}$ & $\mathrm{~N}$ & \multirow{2}{*}{1847} & 9150 & 5093 & 1533 & 32100 \\
\hline 8 & $f_{a x}=R_{a x} /\left(d \cdot \ell_{e f}\right)$ & $\mathrm{MPa}$ & & 19 & 3,44 & 8,84 & 33,6 \\
\hline
\end{tabular}

In Abb. 4 sind die Abhängigkeiten zwischen den Werten derjenigen Merkmale dargestellt, die als erklärende Variable in den folgenden Regressionsrechnungen den größten Beitrag liefern. Die entsprechenden Bestimmtheitsmaße $r^{2}$ sind rechts unten in den Diagrammen angegeben. Die Varianzinflationsfaktoren (VIF) in 
Tabelle 2, die sich ergeben, wenn für jede dieser erklärenden Variablen eine Regression auf jeweils die anderen erklärenden Variablen durchgeführt wird, liegen deutlich unter 10. Sie belegen damit weitgehende lineare Unabhängigkeit (vgl. Chatterjee und Price 1995) zwischen den Werten der Merkmale Nenndurchmesser, Einschraubtiefe und Rohdichte.
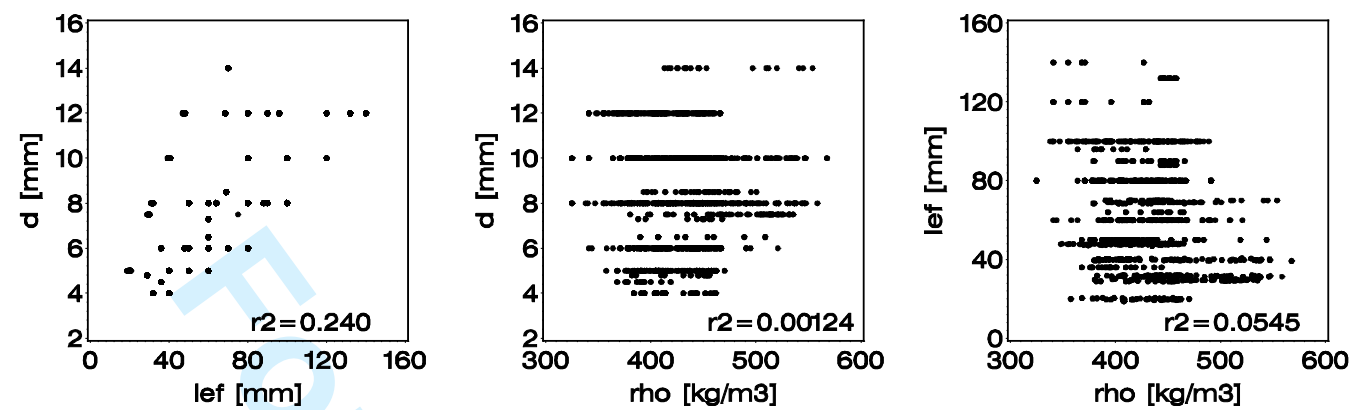

Abb. 4 Streudiagramme: Abhängigkeiten zwischen den Werten der Merkmale Nenndurchmesser, Rohdichte und Einschraubtiefe

Fig. 4 Scatter plots: dependencies between the values of the parameters nominal diameter, softwood density and penetration depth

Tabelle 2 Varianzinflationsfaktoren (VIF); sie zeigen, dass die entscheidenden erklärenden Variablen untereinander weitgehend linear unabhängig sind

Table 2 Variance inflation factors (VIF) showing that there is no collinearity among the crucial explanatory variables

\begin{tabular}{|c|c|c|c|}
\hline Regression von & $\mathrm{N}$ & $\mathrm{r}^{2}$ & $\mathrm{VIF}=1 /\left(1-\mathrm{r}^{2}\right)$ \\
\hline \multirow{2nny}{*}{ auf $\ell_{e f}$ und $\rho$} & \multirow{3}{*}{1847} & 0,264 & 1,36 \\
\cline { 1 - 3 }$\ell_{e f}$ auf $d$ und $\rho$ & & 0,303 & 1,43 \\
\cline { 1 - 3 }$\rho$ auf $d$ und $\ell_{e f}$ & & 0,084 & 1,09 \\
\hline
\end{tabular}

\subsection{Identifikation statistisch signifikanter Modelle}

Ansatzfunktion ist hier eine ganzrationale Funktion mehrerer Veränderlicher 2. Grades. Unabhängige Terme sind darin nicht nur die Variablen in Tabelle 1 Zeile eins bis sechs, linear und quadratisch, sondern auch ihre Kreuzprodukte, jedoch ohne das Produkt $d \cdot d_{k} / d$. Insgesamt sind dies 26 Terme. Regressionen des logarithmierten Ausziehwiderstands $\ln \left(R_{a x}\right)$ bzw. des logarithmierten 
Ausziehparameters $\ln \left(f_{a x}\right)$ auf diese Terme, bei denen die Anzahl der Terme im Modell schrittweise von eins auf 25 erhöht wurde, lieferte das Ergebnis in Tabelle 3. Darin sind auszugsweise für ein bis drei Terme im Modell jeweils die drei Kombinationen mit dem höchsten Bestimmtheitsmaß $\left(\mathrm{r}^{2}\right)$ der Größe nach gestaffelt angegeben, für vier Terme jeweils die beste Kombination. Diejenigen Modelle, die für weitere Betrachtungen ausgewählt wurden, sind mit Pfeilen gekennzeichnet. Als erklärende Variable sind sich erwartungsgemäß der Nenndurchmesser $d$ und der Gewinde-Innendurchmesser $d_{k}$ nahezu ebenbürtig. Es gibt keine Anzeichen dafür, dass der Einschraubwinkel $\alpha$ im Wertebereich [45; $90^{\circ}$ ] einen bedeutenden Beitrag in einem Modell mit überschaubarer Anzahl Terme leistet. In der letzten Zeile der Tabelle 3 sind jeweils noch die höchsten Bestimmtheitsmaße für Modelle mit 25 Termen angegeben. Damit wird deutlich, dass die Beschränkung auf drei bzw. zwei Terme im Modell mechanisch schlüssig und für praktische Anwendungen befriedigend ist. Logarithmieren der Regressanden $R_{a x}$ bzw. $f_{a x}$ bereinigt - was später noch gezeigt wird - die vorliegende nicht konstante Varianz der Fehler (Chatterjee und Price 1995).

Tabelle $3 \quad$ Modellauswahl, Anzahl der Beobachtungen 1847

Table $3 \quad$ Model selection, number of observations used 1847

\begin{tabular}{|c|c|c|c|c|}
\hline & \multicolumn{2}{|c|}{ Regression von $\ln \left(R_{a x}\right)$} & \multicolumn{2}{|c|}{ Regression von $\ln \left(f_{a x}\right)$} \\
\hline $\begin{array}{c}\text { Anzahl Terme } \\
\text { im Modell }\end{array}$ & $\mathrm{r}^{2}$ & $\begin{array}{c}\text { Auswahl } \\
\text { Terme }\end{array}$ & $\mathrm{r}^{2}$ & $\begin{array}{c}\text { Auswahl } \\
\text { Terme }\end{array}$ \\
\hline 1 & $\begin{array}{l}0,825 \\
0,821 \\
0,811 \\
\end{array}$ & $\begin{array}{l}\ell_{e f} \cdot \rho \\
d_{k} \cdot \ell_{e f} \\
d \cdot \ell_{e f} \\
\end{array}$ & $\begin{array}{l}0,263 \\
0,249 \\
0,241 \\
\end{array}$ & $\begin{array}{c}d \\
d^{2} \\
d_{k} \cdot \ell_{e f}\end{array}$ \\
\hline 2 & $\begin{array}{l}0,920 \\
0,916 \\
0,913 \\
\end{array}$ & $\begin{array}{r}d, \ell_{e f} \cdot \rho \\
\ell_{e f}, d \cdot \rho \\
d_{k}, \ell_{e f} \cdot \rho\end{array}$ & $\begin{array}{c}\rightarrow 0,458 \\
0,455 \\
0,452 \\
\end{array}$ & $\begin{array}{c}d, \rho \\
\rho, d \cdot \rho \\
d, \rho^{2}\end{array}$ \\
\hline 3 & $\begin{array}{c}\rightarrow 0,942 \\
0,937 \\
0,935 \\
\end{array}$ & $\begin{array}{c}\ell_{e f}, d \cdot \rho, \ell_{e f}^{2} \\
\ell_{e f}, d_{k} \cdot \rho, \ell_{e f}^{2} \\
\ell_{e f}, d \cdot \ell_{e f}, d \cdot \rho\end{array}$ & $\begin{array}{l}0,481 \\
0,475 \\
0,475 \\
\end{array}$ & $\begin{array}{c}d, \rho, d^{2} \\
d, d^{2}, \rho^{2} \\
d_{k}, \rho, d_{k}^{2}\end{array}$ \\
\hline 4 & 0,945 & $\ell_{e f}, d, \rho, \ell_{e f}^{2}$ & 0,490 & $d, \rho, \alpha \cdot \rho, d^{2}$ \\
\hline 25 & 0,954 & alle Terme $^{\mathrm{a}}$ & 0,555 & alle Terme ${ }^{\mathrm{b}}$ \\
\hline
\end{tabular}




\subsubsection{Regression von $\ln \left(R_{a x}\right)$}

Durch das Ausschließen von Beobachtungen mit normierten Fehlern $e$ außerhalb $[-3 ; 3]$ wurde nach einer Vorab-Regression von $\ln \left(R_{a x}\right)$ auf $\ell_{e f}, d \cdot \rho$ und $\left(\ell_{e f}\right)^{2}$ die Anzahl Beobachtungen von 1847 auf 1838 reduziert. Mithilfe des Ausschlusskriteriums lel > 3 (vgl. Hartung et al. 2005) sollen einige wenige Messwerte, die sich z. B. durch Unregelmäßigkeiten im Versuch ergaben, unberücksichtigt bleiben. Das bedingt eine etwas geringere Streuung der Fehler. Gleichung (5) spezifiziert den funktionalen Zusammenhang für die endgültige Regression. Das Bestimmtheitsmaß $\mathrm{r}^{2}$ beträgt schließlich 0,945, die Standardabweichung der Fehler e 0,1365. In Abb. 5 sind beobachtete Werte in Abhängigkeit von Vorhersagewerten nach Gleichung (5) dargestellt: In Teilbild a für logarithmierte und in $\mathrm{b}$ für wahre Werte. Teilbild a zeigt, dass die Varianz der Fehler $e$ unabhängig ist von der Größe der logarithmierten Vorhersagewerte. Ihre Streuung liegt normalverteilt, vgl. Abb. 6, zwischen den eingezeichneten 99\%Vertrauensgrenzen. Die Voraussetzungen für die Anwendung der KleinsteQuadrate-Methode zur Schätzung der Koeffizienten in Gleichung (5) sind also erfüllt. Teilbild b verdeutlicht die nicht konstante Varianz bei den wahren Werten. Die Punkte sind trichterförmig um die Winkelhalbierende (=Regressionsgerade) angeordnet. Logarithmieren ist hier folglich eine geeignete varianzstabilisierende Maßnahme. Die individuelle Prüfkörperrohdichte ist erklärende Variable in Gleichung (5). Die Gleichung eignet sich daher in besonderen Fällen, bei denen die Rohdichte der Hölzer aus Messungen bekannt ist, zur individuellen Abschätzung von $R_{a x}$.

$$
\begin{aligned}
& \ln \left(R_{a x}\right)=6,739+0,03257 \cdot \ell_{e f}+2,148 \cdot 10^{-4} \cdot d \cdot \rho-1,171 \cdot 10^{-4} \cdot \ell_{\text {lef }}^{2}+e \\
& n=1838 \quad \mathrm{r}^{2}=0,945 \quad e: \mathrm{N}(0 ; 0,1365)
\end{aligned}
$$


a)

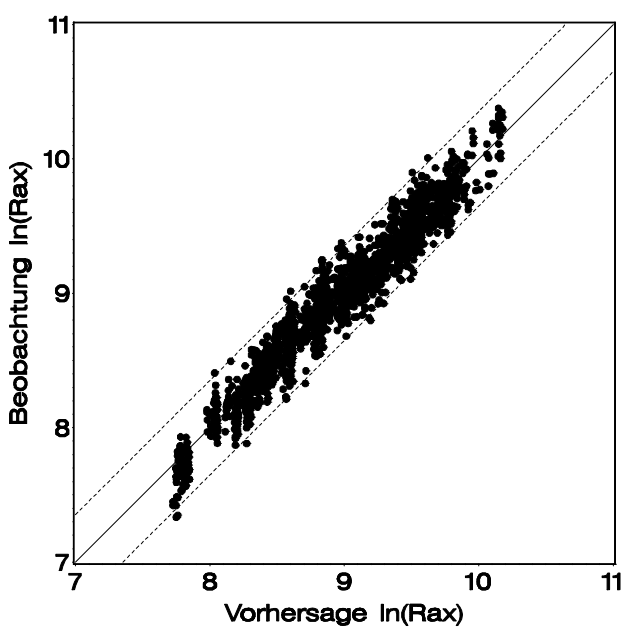

b)

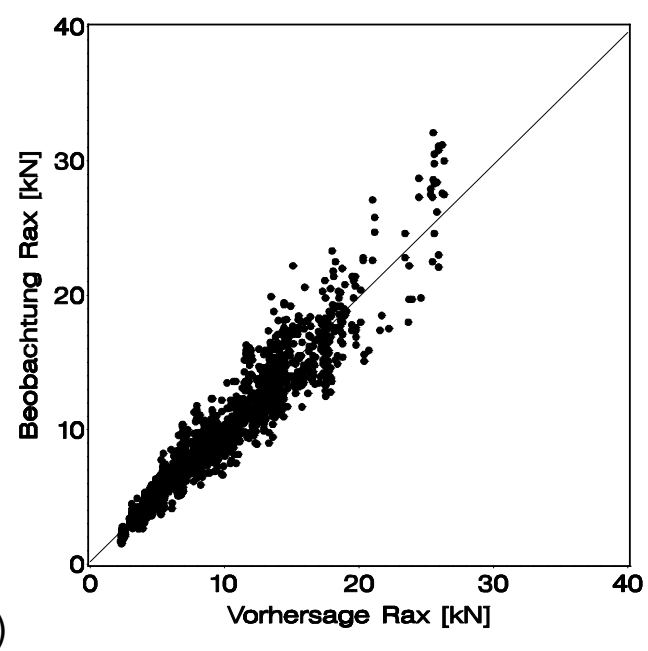

Abb. 5 Regressionsgleichung (5): Beobachteter Ausziehwiderstand in Abhängigkeit vom Vorhersagewert;a) logarithmierte und b) wahre Werte

Fig. 5 Regression equation (5): experimental and predicted values for the axial withdrawal resistance; a) logarithmic and b) true values

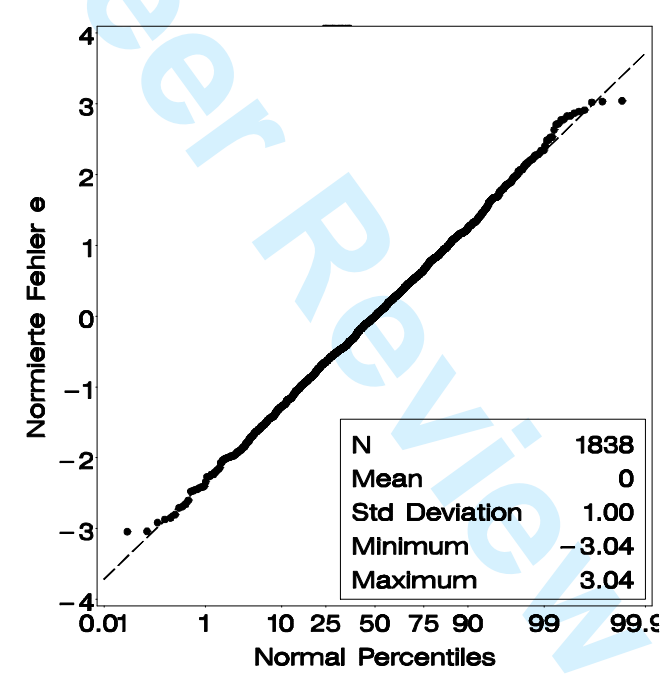

Abb. 6 Regressionsgleichung (5): standardnormalverteilte Fehler

Fig. 6 Regression equation (5): standard normal distributed errors

\subsubsection{Regression von $\ln \left(f_{a x}\right)$}

Gleichung (6) spezifiziert den funktionalen Zusammenhang der endgültigen Regression von logarithmiertem Ausziehparameter $\ln \left(f_{a x}\right)$ auf $d$ und $\rho$. Entsprechend der Herleitung von Gleichung (5) sind die reduzierte Anzahl der Beobachtungen 1839 und Abb. 7 zu verstehen. Die Voraussetzung normalverteilter Fehler ist erfüllt, vgl. Abb. 8, konstante Varianz der Fehler $e$ gilt 
nur mit Einschränkung: Für kleine Vorhersagewerte ist die Streuung etwas geringer als für hohe. Damit ist die Grundvoraussetzung der konstanten Fehlervarianz für die Kleinste-Quadrate-Methode verletzt. Das Bestimmtheitsmaß 0,466 ist möglicherweise leicht verzerrt. Auch in diesem Modell ist die individuelle Prüfkörperrohdichte erklärende Variable.

$$
\begin{aligned}
& \ln \left(f_{a x}\right)=2,359-0,04172 \cdot d+2,039 \cdot 10^{-3} \cdot \rho+e \\
& n=1839 \quad \mathrm{r}^{2}=0,466 \quad e: \mathrm{N}(0 ; 0,1331)
\end{aligned}
$$

\subsection{Modelle in Abhängigkeit von der charakteristischen Rohdichte}

In den beiden Regressionsgleichungen (5) und (6) ist die individuelle Prüfkörperrohdichte, auf etwa 12\% Holzfeuchte bezogen, erklärende Variable. Die Fehler $e$ sind normalverteilt.

a)

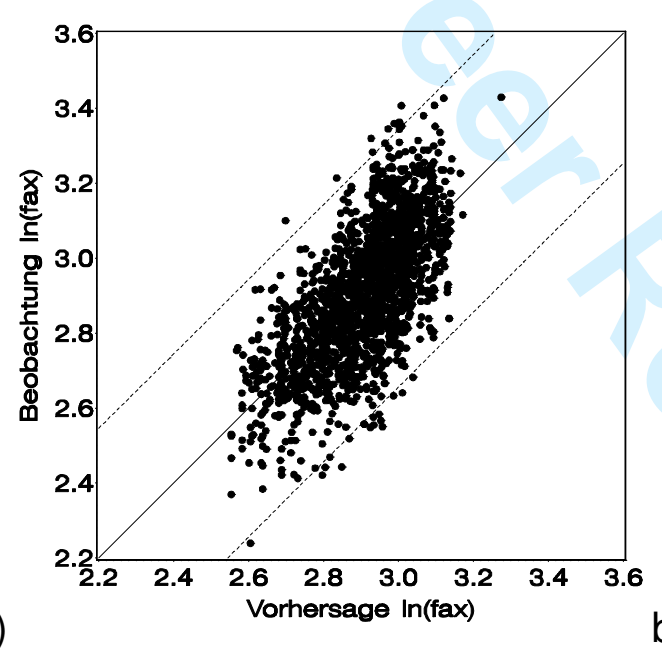

b)

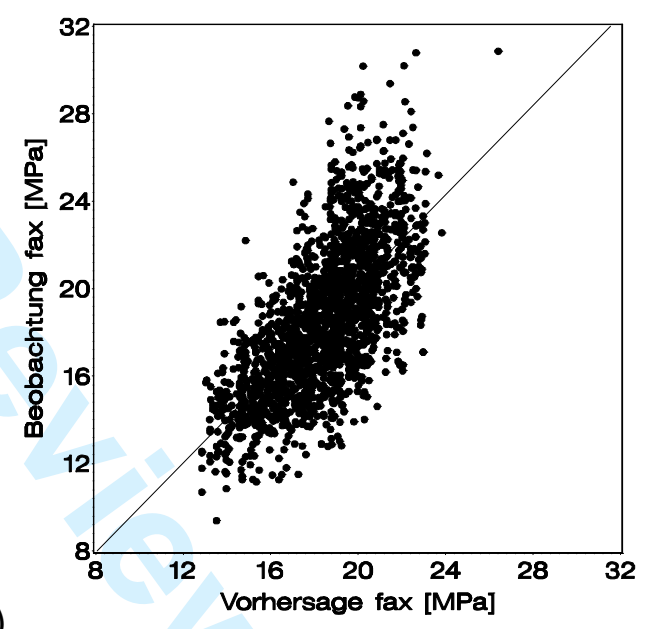

Abb. 7 Regressionsgleichung (6): beobachteter Ausziehparameter in Abhängigkeit vom Vorhersagewert; a) logarithmierte und b) wahre Werte

Fig. 7 Regression equation (6): experimental and predicted value for the axial withdrawal parameter; a) logarithmic and b) true values 


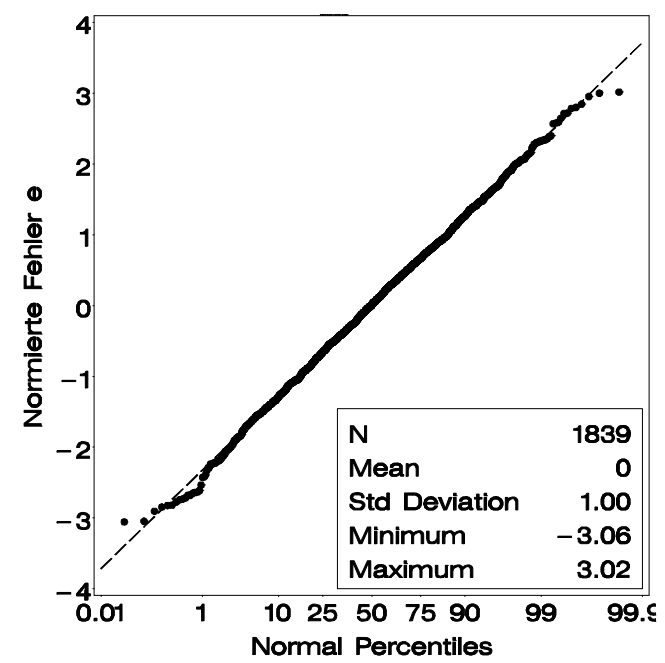

Abb. 8 Regressionsgleichung (6): standardnormalverteilte Fehler

Fig. 8 Regression equation (6): standard normal distributed errors

Für praktische Anwendungen wie Bemessungsaufgaben ist jedoch eine Abhängigkeit von der charakteristischen Rohdichte $\rho_{k}$ erforderlich. Beide Gleichungen sind auf Grund der weitgehend konstanten Varianz ihrer Fehler $e$ uneingeschränkt geeignet, Ausziehwiderstand bzw. -parameter empirisch zu repräsentieren. Mit simulierten Rohdichtewerten für ausgewählte Festigkeitsklassen und simulierten Fehlern soll daher die Abhängigkeit von der charakteristischen Rohdichte numerisch bestimmt werden. Zu diesem Zweck wurden mit beiden Modellen, (5) und (6), für die sechs Festigkeitsklassen C14, C18, C24, C30, C40 und C50 aus EN 338 jeweils $42 d-\ell_{e f}$-Kombinationen untersucht. Die sechs mal sieben Kombinationen setzen sich aus den Elementen gemäß Definition (7) zusammen. Das entspricht insgesamt 252 Untersuchungen je Modell. Bei jeder Untersuchung wurden $1000 R_{a x}$ - bzw. $f_{a x}$-Werte mittels normalverteilter Rohdichtewerte bzw. Fehler simuliert. Die Zuordnungen (8) und (9) zeigen die verwendeten Normalverteilungen. Selbstverständlich wäre beim Modell (6) nur eine Untersuchung unterschiedlicher Nenndurchmesser ausreichend, weil die Einschraubtiefe $\ell_{e f}$ im Modell (6) keine erklärende Variable ist. Die Verwendung der gleichen 42 Kombinationen führt lediglich zu einer Vervielfachung der simulierten Werte je Nenndurchmesser. Das Ergebnis wird dadurch jedoch nicht verfälscht.

$$
\begin{aligned}
& d=\{4,6,8,10,12,14[\mathrm{~mm}]\} \\
& \ell_{e f}=\{20,40,60,80,100,120,140[\mathrm{~mm}]\}
\end{aligned}
$$


$\mathrm{C} 14 \rho: \mathrm{N}(350 ; 36,4)$

$\mathrm{C} 18 \rho: \mathrm{N}(380 ; 36,4)$

$\mathrm{C} 24 \rho: \mathrm{N}(420 ; 42,4)$

$\mathrm{C} 30 \rho: \mathrm{N}(460 ; 48,5)$

$\mathrm{C} 40 \rho: \mathrm{N}(500 ; 48,5)$

$\mathrm{C} 50 \rho: \mathrm{N}(550 ; 54,5)$

$R_{a x, k} \quad e: \mathrm{N}(0 ; 0,1365)$

$f_{a x, k} \quad e: \mathrm{N}(0 ; 0,1331)$

Aus den 1000 simulierten Einzelwerten wurde jeweils der charakteristische Wert, $R_{a x, k}$ bzw. $f_{a x, k}$, ermittelt. In Abb. 9 sind beispielhaft die Verteilungen der simulierten Einzelwerte $R_{a x}$ bzw. $f_{a x}$ für die Festigkeitsklasse C24, für einen Nenndurchmesser von $8 \mathrm{~mm}$ und für eine Einschraubtiefe von $60 \mathrm{~mm}$ (nur erforderlich bei $R_{a x, k}$ ) dargestellt. Die Verteilungen in den Teilbildern a und b gleichen erwartungsgemäß einer Normalverteilung. Insgesamt lagen je Modell 252 simulierte charakteristische Werte des Ausziehwiderstands $R_{a x, k}$ bzw. parameters $f_{a x, k}$ für unterschiedliche $d-\ell_{\mathrm{ef}}$-Kombinationen und unterschiedliche Festigkeitsklassen vor. Eine erneute Regression, und zwar von den simulierten charakteristischen Werten auf die Variablen in den Gleichungen (5) und (6), allerdings nun mit $\rho_{k}$, löst die Modelle von den Fehlertermen und stellt die gewünschte Abhängigkeit von der charakteristischen Rohdichte her. Für den Ausziehwiderstand folgt damit:

$$
\ln \left(R_{a x, k}\right)=6,54+\ell_{e f} \cdot\left(0,03265-1,173 \cdot 10^{-4} \cdot \ell_{e f}\right)+2,35 \cdot 10^{-4} \cdot d \cdot \rho_{k}
$$

und für den Ausziehparameter entsprechend:

$$
\ln \left(f_{a x, k}\right)=2,182-0,04175 \cdot d+2,21 \cdot 10^{-3} \cdot \rho_{k} \text {. }
$$

Die Beziehungen zwischen den simulierten charakteristischen Werten $R_{a x, k} \mathrm{bzw}$. $f_{a x, k}$ sowie den Vorhersagewerten nach den Gleichungen (10) bzw. (11) sind in Abb. 10 dargestellt. Die simulierten charakteristischen Werte lassen sich mit diesen beiden Gleichungen nahezu perfekt beschreiben. Die Bestimmtheitsmaße liegen bei 1,000 bzw. 0,997. So ist es schließlich möglich, den charakteristischen 
Ausziehwiderstand bzw. - parameter für beliebige Festigkeitsklassen abzuschätzen.
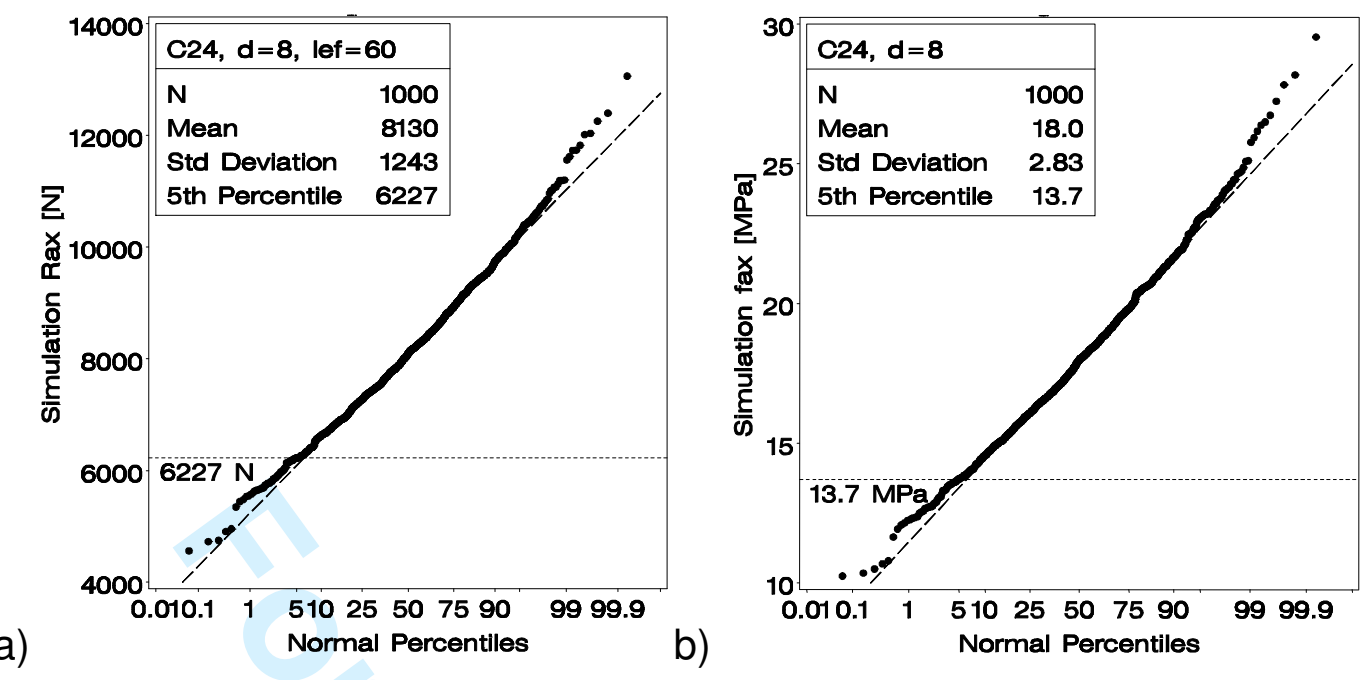

Abb. 9 Simulation für a) $R_{a x}$ und b) $f_{a x}$; aus den Verteilungen kann beispielhaft der charakteristische Wert $R_{a x, k}$ bzw. $f_{a x, k}$ für die Festigkeitsklasse C24 und die $d-\ell_{e f}$-Kombination 8/60 bzw. 8 ermittelt werden.

Fig. 9 Simulation results for a) $R_{a x}$ and b) $f_{a x}$; the characteristic value $R_{a x, k}$ and $f_{a x, k}$, respectively, can be exemplarily determined from the distributions. Values refer to strength class $\mathrm{C} 24$ and $d-\ell_{\mathrm{ef}}-$ combination $8 / 60$ and 8 , respectively.

a)

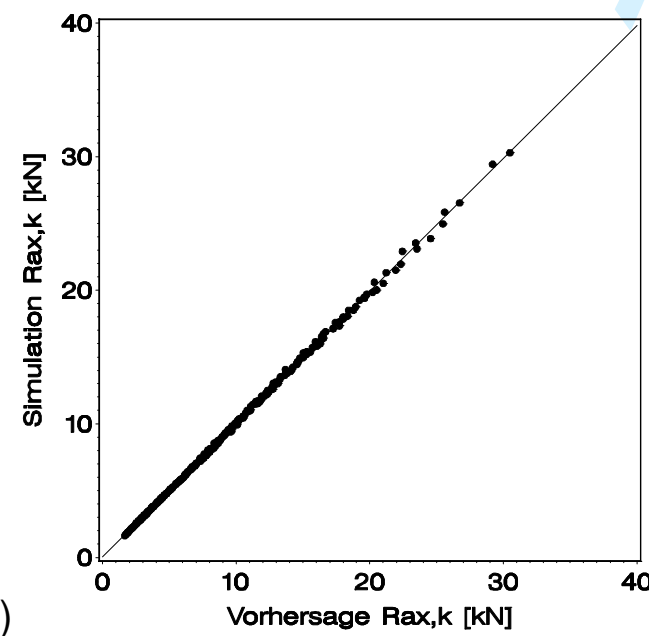

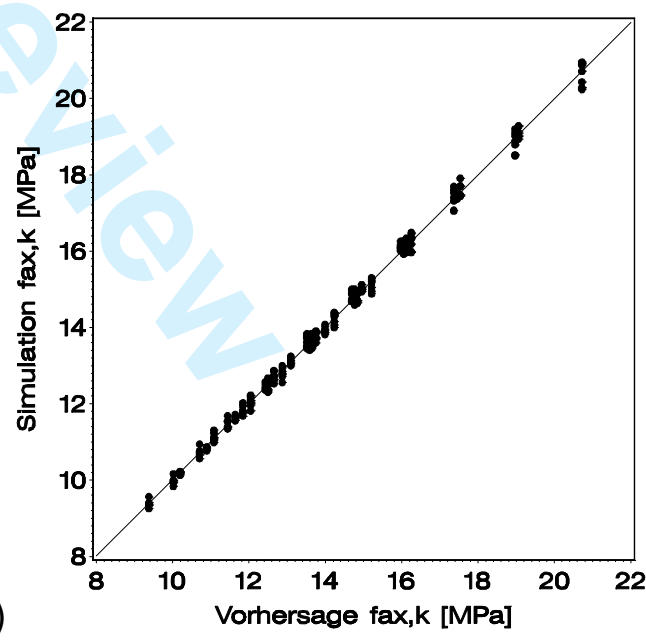

Abb. 10 Regressionsgleichung (10) bzw. (11): a) simulierter charakteristischer Ausziehwiderstand und b) simulierter Ausziehparameter jeweils in Abhängigkeit vom Vorhersagewert

Fig. 10 Regression equations (10) and (11): simulated and predicted value for a) the axial withdrawal resistance and b) the axial withdrawal parameter 


\subsection{Verwendung einer herkömmlichen nichtlinearen Ansatzfunktion}

Es sei noch ein sehr einfacher Ansatz (12) verfolgt, der in der praktischen Anwendung wenige Rechenoperationen erfordert, um den Ausziehparameter $f_{a x}$ zu ermitteln. Er orientiert sich an Gleichung (4), jedoch ohne die Einschraubtiefe $\ell_{e f}$. Diese muss natürlich bei einer Bemessungsaufgabe in einem weiteren Schritt zusammen mit dem Nenndurchmesser $d$ - mit dem Ausziehparameter $f_{a x}$ multipliziert werden.

$$
f_{a x}=A \cdot \rho \cdot d^{B}
$$

Eine nichtlineare Regression von $f_{a x}$ auf $\rho$ und $d$ mit 1830 Beobachtungen, also 17 Beobachtungen mit normierten Fehlern $e$ außerhalb [-3,3] nach einer VorabRegression ausgeschlossen, liefert den folgenden Zusammenhang:

$$
\begin{aligned}
& f_{a x}=0,0857 \cdot \rho \cdot d^{-0,3423}+e, \\
& n=1830 \quad \mathrm{r}^{2}=0,484 \quad e: \mathrm{N}(0 ; 2,388) .
\end{aligned}
$$

Dieses Modell ähnelt sehr demjenigen in Abschnitt 2.2.2. Auch hier ist die Varianz der Fehler, vgl. Abb. 11 Teilbild a, nur näherungsweise konstant, das Bestimmtheitsmaß möglicherweise leicht verzerrt. Die Fehler $e$ sind annähernd normalverteilt, siehe Abb. 11 Teilbild b.

a)

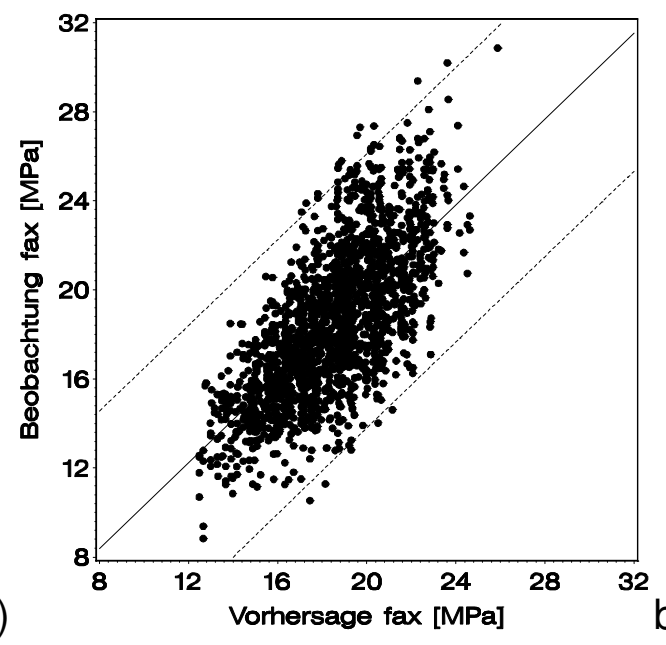

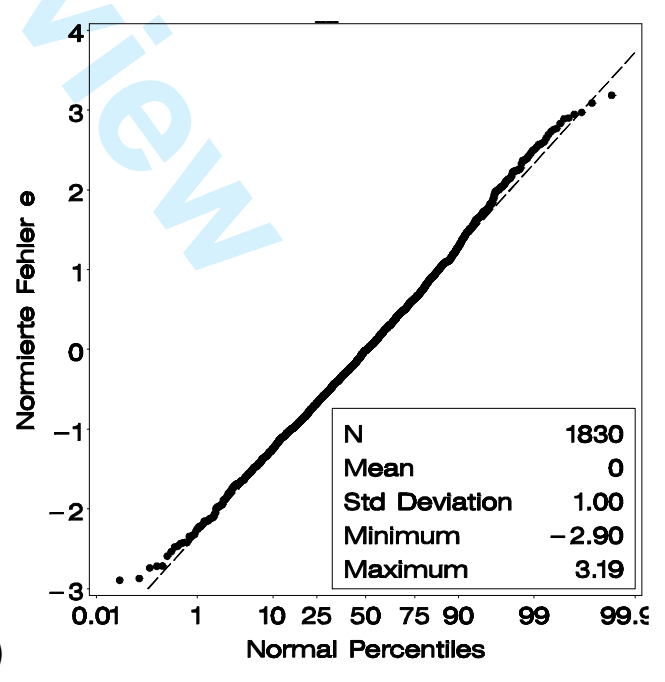

Abb. 11 Regressionsgleichung (13): beobachteter Ausziehparameter in Abhängigkeit vom a)Vorhersagewert und b) standardnormalverteilten Fehlern

Fig. 11 Regression equation (13): experimental and predicted value for the a) axial withdrawal parameter and b) standard normal distributed errors 
Entsprechend dem Simulationsverfahren in Abschnitt 2.3 werden der Koeffizient und Exponent in Beziehung (13) so angepasst, dass die charakteristische Rohdichte $\rho_{k}$ erklärende Variable ist. Dann gilt für den charakteristischen Ausziehparameter:

$$
f_{a x, k}=0,0872 \cdot \rho_{k} \cdot d^{-0,4119} \text {. }
$$

Die Güte der Regression von simulierten charakteristischen Ausziehparametern auf die charakteristische Rohdichte (der sechs untersuchten Festigkeitsklassen) und unterschiedliche Nenndurchmesser verdeutlicht Abb. 12.

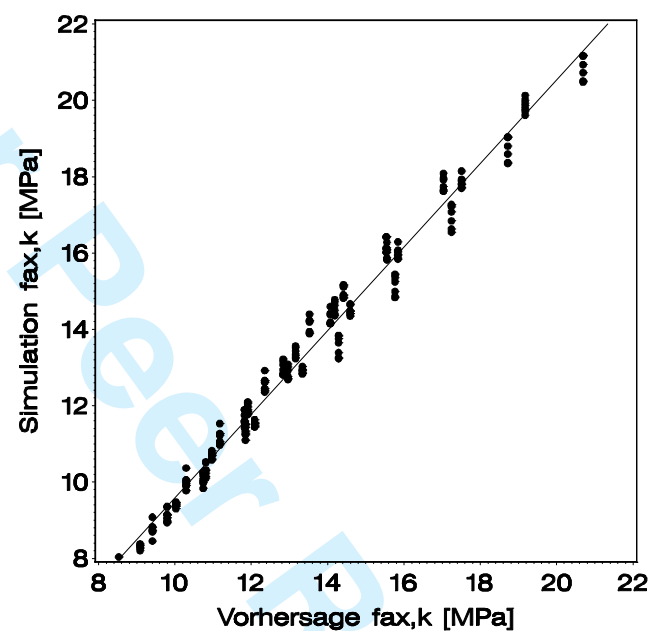

Abb. 12 Regressionsgleichung (14): simulierter charakteristischer Ausziehparameter in Abhängigkeit vom Vorhersagewert

Fig. 12 Regression equation (14): simulated and predicted value for the characteristic axial withdrawal parameter

\section{Bewertung der Modelle}

Für eine gemeinsame Bewertung der Modelle (10), (11) und (14) seien ihre Vorhersagewerte $R_{a x, k}$ für 18 unterschiedliche $d-\ell_{e f}$-Kombinationen untereinander verglichen. Es gelten dafür die Vereinbarungen: Werte des Modells (10) dienen als Basis, auf die die $R_{a x, k}$-Werte der Modelle (11) und (14) bezogen werden. Für Vergleiche dienen dann die Quotienten (15) und (16). Die 18 ausgewerteten Kombinationen sind in Tabelle 4 zusammengestellt. Einer geringeren Einschraubtiefe $\ell_{e f}$ bei kleinen Nenndurchmessern und umgekehrt wird dabei Rechnung getragen. Baupraktische Anwendungen seien damit abgedeckt. 
Betrachtet werden die Festigkeitsklassen C24 und C40 mit charakteristischen Rohdichten von 350 bzw. $420 \mathrm{~kg} / \mathrm{m}^{3}$.

In Abb. 13 sind die Quotienten gegenübergestellt. Es wird deutlich: Die Modelle (11) und (14) führen auf ähnliche Werte, die aber im Mittel nur 92\% der Werte des Modells (10) erreichen. Gute Übereinstimmung zwischen allen Modellen besteht für Nenndurchmesser von 9 mm, also die Kombinationen 4-6 und 13-15. Hinsichtlich einer vergleichsweise hohen Ausziehtragfähigkeit ist Modell (10) zu bevorzugen.

$$
\begin{gathered}
\eta_{11 / 10}=\frac{R_{a x, k} \text { Gl.(11) }}{R_{a x, k} \text { Gl.(10) }} \\
\eta_{14 / 10}=\frac{R_{a x, k} \text { Gl.(14) }}{R_{a x, k} \text { Gl.(10) }}
\end{gathered}
$$

Tabelle $4 \quad d-\ell_{e f}$-Kombinationen und Rohdichte für Modellvergleiche

Table $4 \quad d-\ell_{e f}$-combinations and characteristic softwood density for comparisons between the models

\begin{tabular}{|c|c|c|c|c|}
\hline Kombination & $d$ in $\mathrm{mm}$ & $\ell_{e f}$ in $\mathrm{mm}$ & $\rho_{k}$ in $\mathrm{kg} / \mathrm{m}^{3}$ & Festigkeitsklasse \\
\hline $1-3$ & 4 & $20-40-60$ & & \multirow{2}{*}{ C24 } \\
\cline { 1 - 3 } $4-6$ & 9 & $40-70-100$ & & \\
\hline $7-9$ & 14 & $60-100-140$ & & \multirow{2}{*}{ C40 } \\
\cline { 1 - 3 } $10-12$ & 4 & $20-4060$ & & \\
\hline $13-15$ & 9 & $40-70-100$ & & \\
\hline $16-18$ & 14 & $60-100-140$ & & \\
\hline
\end{tabular}




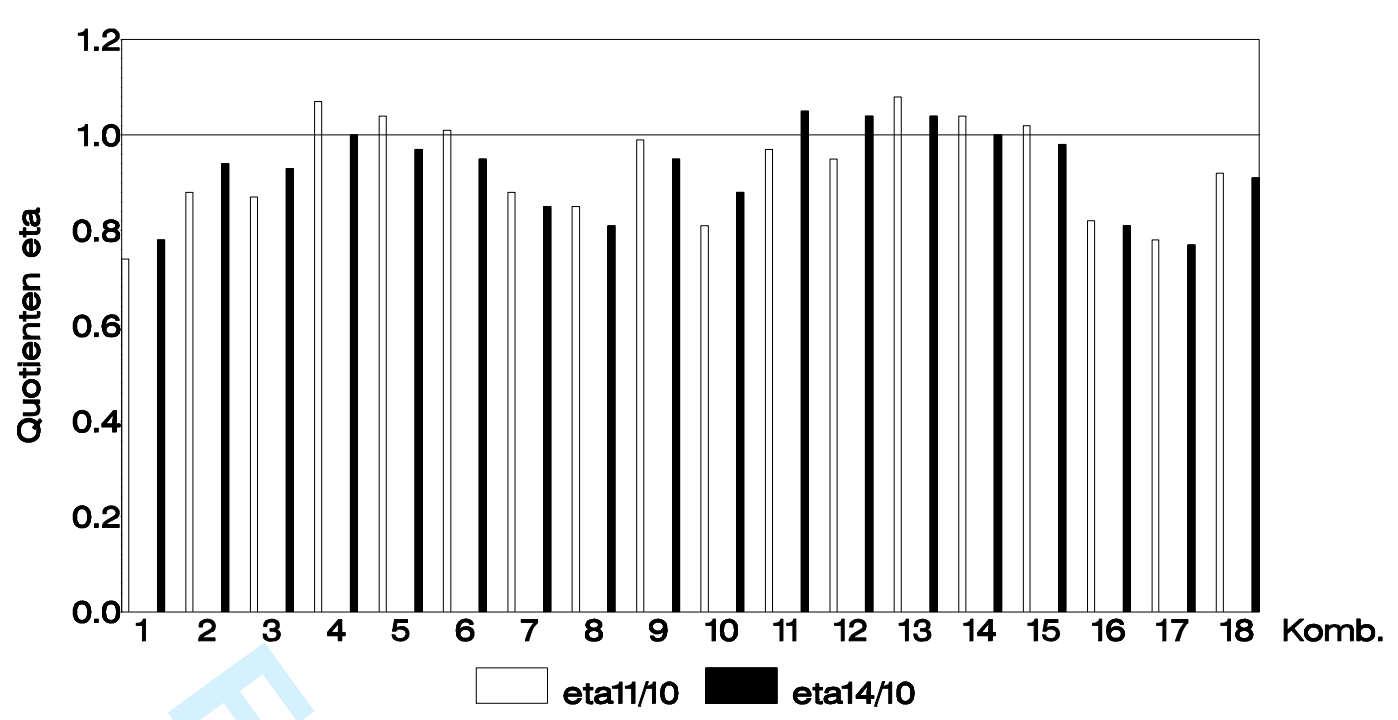

Abb. 13 Vergleich Modelle (11) und (14) mit Modell (10) mittels Vorhersagewerten

Fig. 13 Model comparison: (11) and (14) with (10) by means of predicted values

Für weitere Vergleiche mit den Berechnungsgleichungen (1), (2) und (3) (aus DIN 1052, Blaß et al. 2006, EN 1995-1-1) sei Modell (10) auf Grund der günstigen Vorhersagewerte weiterhin die Vergleichsbasis. Die entsprechenden Quotienten werden dann mit den Gleichungen (17) bis (19) berechnet, und zwar für die gleichen 18 Kombinationen in Tabelle 4. Der Einschraubwinkel $\alpha$ sei $90^{\circ}$. Die Vorhersagewerte nach Gleichung (1) werden mit TFK 3 berechnet. Die zulässigen Nenndurchmesser für Gleichung (3) werden um 2 mm unter- bzw. überschritten. Die Gegenüberstellung der Quotienten zeigt Abb. 14: Im Mittel erreichen die Werte nach Gleichung (1) nur zu 78\% das Niveau der Werte nach Gleichung (10), bei Gleichung (2) sind es immerhin 93\% und bei Gleichung (3) nur 76\%. Teilweise betragen die Quotienten etwa 50\%. Würde die Auswertung in Abb. 14 für den Einschraubwinkel $\alpha=45^{\circ}$ durchgeführt werden, wären die prozentualen Verhältnisse um weitere $10 \%$ kleiner.

$$
\begin{aligned}
& \eta_{1 / 10}=\frac{R_{a x, k} \text { Gl.(1) }}{R_{a x, k} \text { Gl.(10) }} \\
& \eta_{2 / 10}=\frac{R_{a x, k} \text { Gl.(2) }}{R_{a x, k} \text { Gl.(10) }} \\
& \eta_{3 / 10}=\frac{R_{a x, k} \text { Gl.(3) }}{R_{a x, k} \text { Gl.(10) }}
\end{aligned}
$$




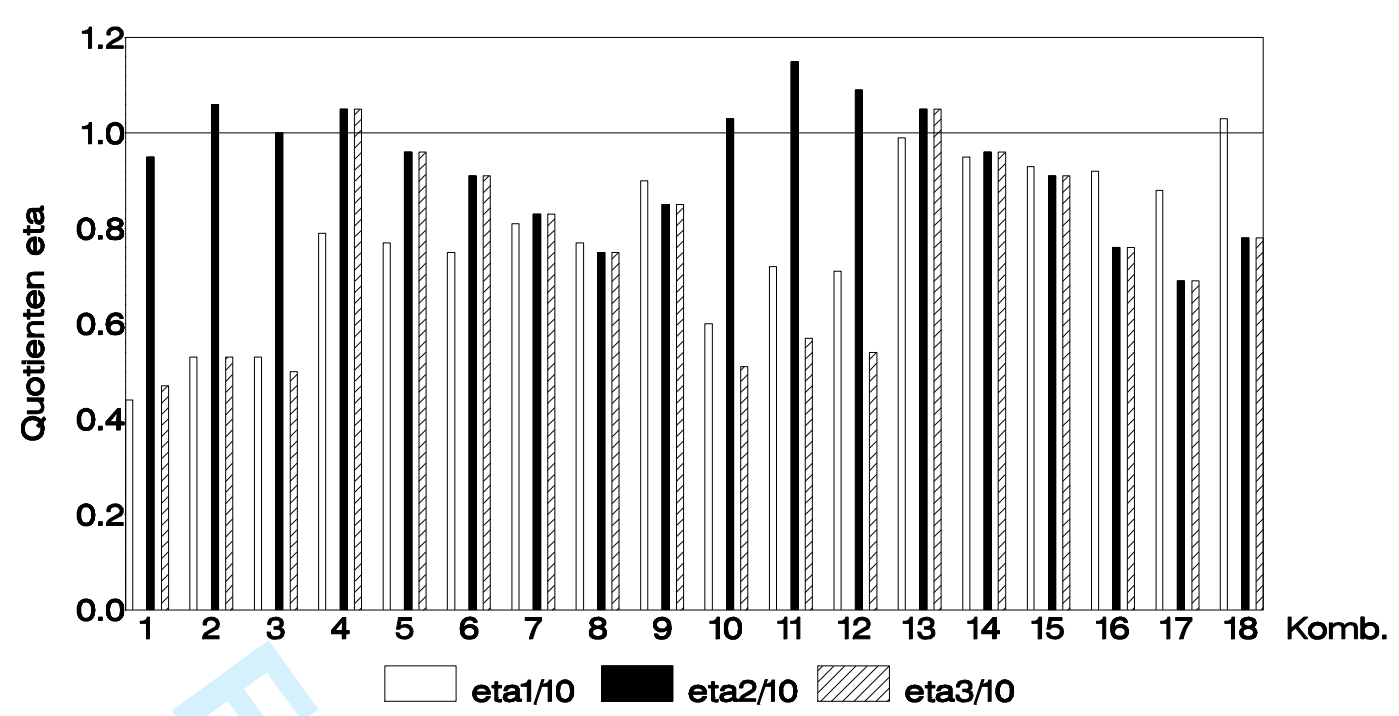

Abb. 14 Vergleich Modelle (1), (2) und (3) mit (10) mittels Vorhersagewerten

Fig. 14 Model comparison: (1), (2) and (3) with (10) by means of predicted values

Die fünf Vergleiche zeigen, dass die direkte Modellierung von $R_{a x, k}$ mit Gleichung (10) zu den höchsten Werten führt. Für die Berechnung ist weder der Einschraubwinkel noch der Ausziehparameter erforderlich. Das macht diese Gleichung ebenso praktisch wie ihre Alternativen. Im Vergleich zu EN 1995-1-1 ist der zulässige Wertebereich für den Nenndurchmesser größer. Die über Simulationen hergeleitete Abhängigkeit von der charakteristischen Rohdichte ist konsistent hinsichtlich der Festigkeitsklassen in EN 338. Das bestärkt die Zuverlässigkeit der Vorhersagewerte für eine weite Spanne der charakteristischen Rohdichte.

\section{Zusammenfassung}

Auf der Grundlage von Ergebnissen aus etwa 1850 Ausziehversuchen mit selbstbohrenden Holzschrauben aus Nadelholz wurden ein Berechnungsmodell für den charakteristischen Ausziehwiderstand und zwei weitere für den Ausziehparameter hergeleitet. Das Modell für den Ausziehwiderstand enthält als unabhängige Variable den Nenndurchmesser, die Einschraubtiefe und die charakteristische Rohdichte einer Festigkeitsklasse in EN 338. In den Modellen für den Ausziehparameter sind es nur der Nenndurchmesser und die charakteristische Rohdichte. 
Das Berechnungsmodell für den Ausziehwiderstand, verglichen mit den beiden Modellen für den Ausziehparameter, führt auf die höchste Ausziehtragfähigkeit. Im Vergleich mit den Berechnungsgleichungen in DIN 1052 und Eurocode 5 sind die Werte dieses Modells im Mittel um 30\% höher. Weitere Kennzeichen: Es gilt für Nenndurchmesser von 4 bis 14 mm, für Einschraubtiefen von 20 bis 140 mm, und ist unabhängig vom Einschraubwinkel zwischen $45^{\circ}$ und $90^{\circ}$ anwendbar. Da in den Ergebnissen der Ausziehversuche unterschiedliche Schraubentypen etlicher Hersteller anteilig erfasst wurden, sind die neuen Modelle geeignet, um die Ausziehtragfähigkeit handelsüblicher selbstbohrender Holzschrauben zu berechnen. Dass das neue Modell für den Ausziehwiderstand eine deutliche Tragfähigkeitssteigerung bedeutet, möge selbstbohrende Holzschrauben für Verbindungen mit schräg angeordneten Schrauben in Zukunft noch attraktiver machen. 


\section{Literatur}

Bejtka I, Blaß HJ (2002) Joints with inclined screws. CIB-W18/35-7-4, Kyoto, Japan

Bejtka I, Blaß HJ (2005) Self-tapping screws as reinforcements in connections with dowel-type fasteners. CIB-W18/38-7-4, Karlsruhe, Germany

Bejtka I, Blaß HJ (2006) Self-tapping screws as reinforcements in beam supports. CIB-W18/39-7-

2, Florence, Italy

Chatterjee S, Price B (1995) Praxis der Regressionsanalyse, 2. Aufl. Oldenbourg Verlag:

München, Wien

Blaß HJ, Bejtka I, Uibel T (2006) Tragfähigkeit von Verbindungen mit selbstbohrenden

Holzschrauben mit Vollgewinde, Bd. 4. Karlsruher Berichte zum Ingenieurholzbau.

Universitätsverlag Karlsruhe, Karlsruhe

Blaß HJ, Schmid M (2001) Self-tapping screws as reinforcement perpendicular to the grain in timber connections. RILEM Symposium Joints in Timber Structures, Stuttgart, Germany:163-172

Hartung J, Elpelt B, Klösener KH (2005) Statistik, 14. Aufl. Oldenbourg Verlag: München, Wien Jönsson J (2005) Load carrying capacity of curved glulam beams reinforced with self-tapping screws. Holz Roh- Werkst 63:342-346

Kevarinmäki A (2002) Joints with inclined screws. CIB-W18/35-7-3, Kyoto, Japan

DIN 96:1986-12 Halbrund-Holzschrauben mit Schlitz

DIN 97:1986-12 Senk-Holzschrauben mit Schlitz

DIN 571:1986-12 Sechskant-Holzschrauben

DIN 1052:2008-12 Entwurf, Berechnung und Bemessung von Holzbauwerken - Allgemeine Bemessungsregeln und Bemessungsregeln für den Hochbau

DIN 7998:1975-02 Gewinde und Schraubenenden für Holzschrauben

EN 338:2003 Bauholz für tragende Zwecke - Festigkeitsklassen

EN 1382:1999 Holzbauwerke - Prüfverfahren - Ausziehtragfähigkeit von Holzverbindungsmitteln EN 1995-1-1:2004+A1:2008 Eurocode 5: Bemessung und Konstruktion von Holzbauteilen - Teil 1-1: Allgemeines - Allgemeine Regeln und Regeln für den Hochbau EN 14592: 2008 Holzbauwerke - Stiftförmige Verbindungsmittel - Anforderungen 